\title{
R-ISSR - Tool for Generation of a New Type of Products, Applied for the Identification of Putative Molecular Marker Linked to QTL Determined Tolerance to Nutrient Deprivation Stress in Rye (Secale cereale L.)
}

\author{
Miłosz SMOLIK \\ West Pomeranian University of Technology in Szczecin, Department of Plant Genetics, Breeding and \\ Biotechnology, Stowackiego 17,71-434 Szczecin,Poland; msmolik@zut.edu.pl
}

\begin{abstract}
This study presents results confirming the possibility of the application of various combinations of RAPD and ISSR primers in one multiplex PCR for the rye genome and generating a new type of R-ISSR products. The plant material consisted of two inbred lines (Ot)-6 and Ot1-3), $F_{1}$ and two bulks (tolerant and susceptible), selected from a population of RILs $\left(F_{9}\right)$ with different responses to stress caused by nutrient deprivation at the seedling stage. In one PCR reaction, the DNA of five genotypes was amplified separately, with RAPD, ISSR, and RAPD+ISSR primers. In total, 28 R-ISSR combinations were tested by combining 20 and 8 appropriately selected RAPD and ISSR primers. 567 loci were amplified, including 230 RAPD, 136 ISSR and 207 amplified R-ISSR loci, of which 114 were new. It was shown that only one ISSR product was identified amongst sequenced R-ISSR products with identical electrophoretic mobility as comigrating RAPDs and ISSRs. The remaining ones had heteroamplicons (R-ISSR) sequences. A similar range of variability was observed both in R-ISSR profiles and in RAPD, as well as in ISSR. The correlation coefficient between the matrices of genetic similarity for five rye genotypes calculated by means of the Mantel test was highly significant $\mathrm{r}_{\mathrm{AB} . \mathrm{C}}=0.964$.
\end{abstract}

Keywords: ISSR, molecular markers, nutrient deficiency, rye, R-ISSR, RAPD

\section{Introduction}

Identification of genes, factors and mechanisms regulating their expression enables their role in the functioning of organisms to be understood (Liu et al., 2008; Moller et al., 2011; Xu et al., 2011). In organic plant cultivation, including cultivation of plants with decreased nutrient requirements, such information may significantly facilitate selection of desired genotypes (Wolfe et al., 2008).

Such research provides a wealth of biological information and it is becoming increasingly easier to gain access to it. It is also increasingly easier to assign significance and sense to biological information obtained within experiments which are conducted based on information already collected using the synteny and collinearity of genomes (Devos and Gale, 2000).

Rye (Secale cereale L.) is an important cereal species grown in many countries of Central, Eastern and Northern Europe (RYE-BELT). Rye is used as a source of resistance genes for many cereal diseases, winter hardiness, tolerance to sprouting, drought, and nutrient deficiencies that can be transferred to closely related cereal crops (Geiger and Miedaner, 2009; Hillel and Rosenzweig, 2005; Wolfe et al., 2008). Compared to wheat and barley, the data available on rye with various sequences collected at gene banks (primarily EST) remains modest ( 10,000 sequences) (Bartoš et al., 2008).
A large part of the rye genome (ca. 92\%) is constituted of microsatellite sequences. Their structure and the mechanisms of their formation were presented in a study by Flavell et al. (1974), while their polymorphisms in studies by Bednarek et al. (2003), Bolibok et al. (2006), Bartoš et al. (2008) and Akhavan et al. (2010).

The essence of R-ISSR lies in the combination of decamer (Williams et al., 1990) and microsatellite (Zietkiewicz et al., 1994) primers in one multiplex PCR. Amplification is performed within new microsatellite and inter-microsatellite DNA regions, which are not amplified separately using RAPD (Ćwiklińska et al., 2010; Myśków et al., 2001) or ISSR (Bolibok et al., 2006; Saleh, 2011; Ye et al., 2005).

This study demonstrates the possibility of the application of the R-ISSR technique as a screening tool to describe the new range of variability in the rye genome. It was devised for rye plant material prepared for research in response to nutrient deprivation stress aimed at: describing generated R-ISSR products, identifying R-ISSR loci for rye, examining the possibility of using them to identify a new type of putative molecular marker linked to different QTL (Quantitative Trait Loci), including those which determine rye tolerance to abiotic stress caused by nutrient deprivation at the seedling stage. 


\section{Materials and methods}

\section{Plant material}

The plant material consisted of two rye inbred lines: Ot0-6 and Ot1-3, hybrid $\mathrm{F}_{1}(\mathrm{Ot} 0-6 \times$ Ot1-3), two bulks (tolerant and susceptible to nutrient deprivation in medium assessed according to the method described by Rzepka-Plevneš and Kurek (1997), twenty tolerant and susceptible RILs $\left(\mathrm{F}_{9}\right)$ from two groups differing in their response to abiotic stress, selected in accordance with the methodology described by Michelmore et al. (1991) (unpublished results).

\section{$D N A$}

DNA was extracted from fresh leaves of 10-day-old seedlings using a DNeasy Plant Mini Kit (Qiagen) and was quantified using a GeneQuant Unit (Pharmacia LKB) to obtain 40-50 ng DNA pro $1 \mu$ l of DNA template.

\section{RAPD and ISSR primers}

A set of twenty selected RAPD and eight selected ISSR primers were used (Tab. 1). Sequences of RAPD and ISSR primers were designed by the University of British Columbia, Canada [RAPD Primer Sets \# 1, 2, 3, 4 and 7; Primer Set \#9 (microsatellite)] and synthesized (each in $0.025 \mathrm{nmol}$ scale) by Sigma-Aldrich.

\section{PCR conditions}

A series of multiplex PCR reactions (in two replications) was conducted on them in order to select RAPD and ISSR primers cooperating in the same thermal profile, i.e. providing distinct fingerprints. In the following stage of the research, the experiment was repeated with the previously selected primers, adding two inbred lines (Ot0-6, Ot 1-3) and hybrid $\mathrm{F}_{1}(\mathrm{Ot} 0-6 \times \mathrm{Ot} 1-3)$ to the bulks. With five such chosen templates, reactions in the same thermal profile were carried out. Finally, electrophoresis was conducted for 15 accessions, five for each of the techniques applied.

\section{DNA amplifications}

DNA amplifications were conducted according to the method described by Williams et al. (1990). The reaction mixture $(25 \mu \mathrm{l})$ contained $10 \times$ PCR buffer with $\left(\mathrm{NH}_{4}\right)_{2} \mathrm{SO}_{4}, 2 \mathrm{mM}$ of dNTPs, $25 \mathrm{mM} \mathrm{MgCl}_{2}, 2.5 \mu \mathrm{M}$ of primer (RAPD, ISSR, RAPD+ISSR, respectively), $1 \mathrm{U}$ of Taq DNA polymerase enzyme (Fermentas) and 50-70 ng of DNA template. The reactions were performed in a Mastercycler 5333 (Eppendorf). The program of thermal cycling was as follows: initial activation step at $95^{\circ} \mathrm{C}$ for 3 min followed by 32 cycles of $30 \mathrm{~s}$ at $94^{\circ} \mathrm{C}, 30 \mathrm{~s}$ at $36^{\circ} \mathrm{C}$ and $2 \mathrm{~min}$ at $72^{\circ} \mathrm{C}$, with a final extension step at $72^{\circ} \mathrm{C}$ for $10 \mathrm{~min}$.

\section{Electrophoresis and data analysis}

PCR products were analyzed by electrophoresis (SubCell GT, Bio-Rad) in $1 \times$ TBE buffer in $1.5 \%$ agarose gel
(Basica LE GQT - Prona). An O'RangeRuler 200 bp DNA Ladder (3000-200 bp) and a GeneRuler 100 bp Plus DNA Ladder (3000-100 bp) (Fermentas) were used as size markers. PCR products were stained with ethidium bromide, visualized (MiniBIS Pro - DNR Bio-Imaging Systems) and scored for band presence or absence. The DNA fingerprints generated by each of the primers or primer pairs employed (Tab. 1) were used to assess genetic variability in the rye genotype tested. Presence (1) or absence (0) of each amplicon was scored for each genotype. Matrices of similarity were generated using PhylTool (Buntjer, 2001) and were compared by calculating the product-moment correlation (Pearson) between matrices and Mantel (Daniel's) test statistics (Z) of significance using XLSTAT. The relationships between the five rye genotypes were shown in the form of a dendrogram produced by cluster analysis using the UPGMA method. The strength of the internal branches from the resulting tree was tested by the TREECON bootstrap analysis application using 2,000 replications (Van de Peer and De Wachter, 1994).

\section{PCR product extraction}

PCR product extraction from the agarose gel was performed in order to allow sequencing using a Qiaquick Gel Extraction Kit (Qiagen, Germany). DNA was prepared from single bands selected from two R-ISSR fingerprints (Fig. 2A and B). DNA was extracted from R-ISSR products, which were assumed to be a putative molecular marker, or 'like'-RAPD or 'like'-ISSR products due to the identical electrophoretic mobility in agarose gels and from two PCR products, which were considered to be the new RISSR loci, according to Ye et al. (2005) and Saleh (2011). They were not observed in RAPD or ISSR profiles.

\section{TOPO TA Cloning}

Products were cloned in a pCR2.1 vector using a TOPO TA cloning kit (Invitrogen), then transformed into TOP 10 competent $E$. coli cells according to the manufacturer's instructions, and the correct transformants were selected according to the standard blue-white screening procedure. Plasmid DNA was purified from a $2 \mathrm{ml} \mathrm{cul-}$ ture of individual colonies using a Plasmid Midi kit (A\&A Biotechnology, Poland). Insert sizes were checked using $1 \%$ agarose electrophoresis after $5 \mathrm{~min} E c o$ RI fast digest restriction (Fermentas).

\section{Sequencing and data analysis}

Sequences were obtained using a Beckman Coulter TDCS kit and run on a CEQ 8000 capillary sequencer according to the manufacturer's instructions. The sequences were edited and assembled into a full-length insert sequence using the BioEdit tool (http://www.mbio.ncsu. edu/BioEdit/bioedit.html) to investigate the presence of a combination of sequences of primers used for the amplifications and were analyzed for homology using the BLASTN and BLASTX algorithms in comparison with the GenBank database. 
240

\section{Results}

Twelve ISSR and thirty RAPD primers were used in multiplex PCR. The reaction was performed for fifty varying combinations of RAPD and ISSR primers. Products visualized on the electrophoregrams with nineteen RAPD and eight ISSR were used for analysing the results.

It was shown that in the DNA fingerprints of the five rye genotypes there is a range of variability enabling the identification of monomorphic, polymorphic and genotype-specific loci. Their presence or absence in the genetic profile was described in various configurations for hybrid $\mathrm{F}_{1}$, bulks (Fig. 1) and, in particular, forty RILs, and significant differences were found between them as regards their response to nutrient deprivation stress.

New genomic R-ISSR loci were amplified by combining RAPD and ISSR primers in one multiplex PCR (Fig. 2).
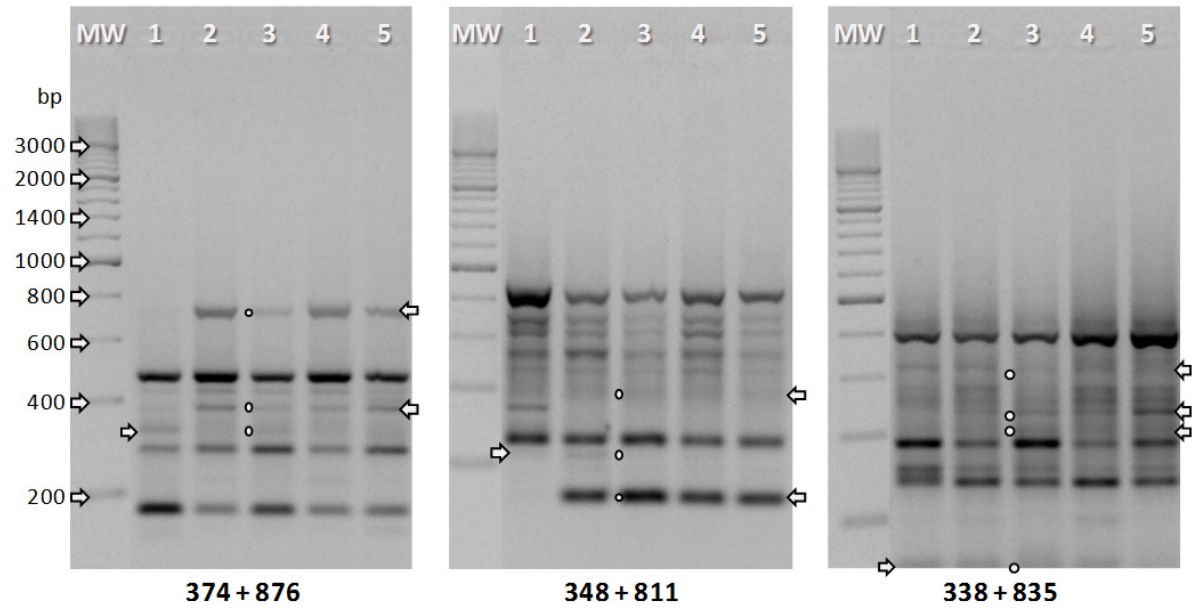

Fig. 1. Electrophoregrams of selected R-ISSR fingerprints, presented loci amplified for five different rye genotypes. White arrows and points mark selected amplicons observed in the $\mathrm{F}_{1}$ profile as co-dominant markers

MW - molecular weight ladder; 1 - Ot0-6; 2 - bulk - RILs tolerant to nutrient deprivation, 3 - $\mathrm{F}_{1}$ (Ot0-6 $\times$ Ot 1-3); 4 - bulk - RILs susceptible to nutrient deprivation, 5 - Ot1-3. Primer - RAPD: 374, 348, 338; ISSR: 876, 811, 835.
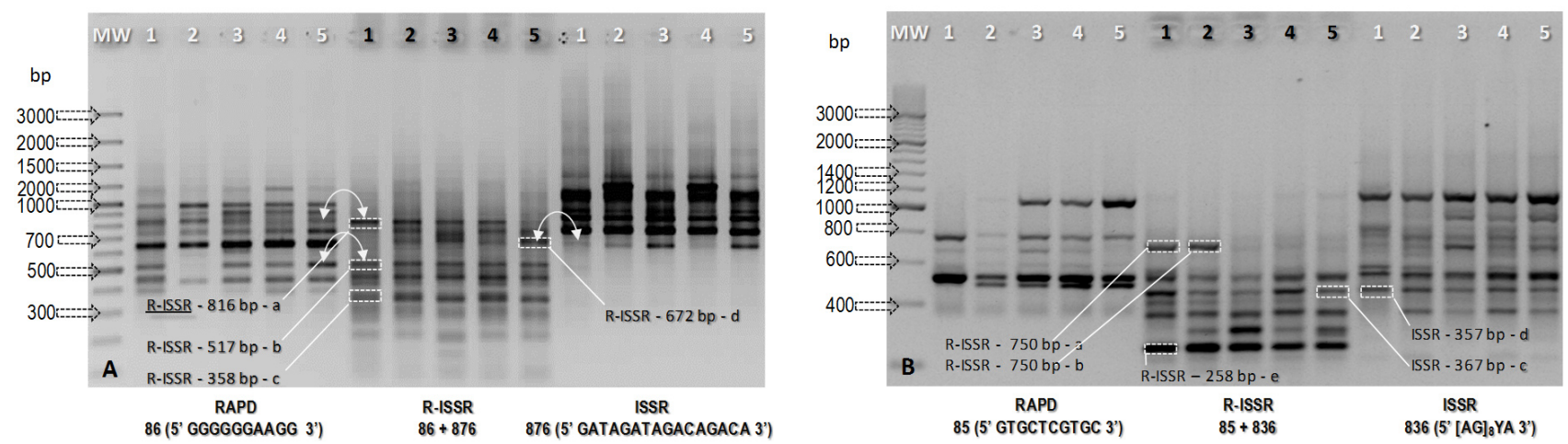

Fig. 2. Fingerprints of RAPD, R-ISSR and ISSR products amplified for five different rye genotypes. White rectangles mark selected amplicons (their length in bp) extracted from the gels, cloned into TOPO vectors and sequenced

MW - molecular weight ladder, 1 - Ot0-6; 2 - bulk - RILs tolerant to nutrient deprivation; 3 - $F_{1}$ (Ot0-6 $\times$ Ot1-3); 4 - bulk - RILs susceptible to nutrient derivation; 5 - Ot1-3 
Tab. 1. Characteristics of RAPD, R-ISSR and ISSR loci amplified for selected genotypes of rye

\begin{tabular}{|c|c|c|c|c|c|c|c|c|c|c|c|c|}
\hline \multicolumn{4}{|c|}{ Primers and sequence } & \multirow{2}{*}{$\begin{array}{l}\text { Range of } \\
\text { amplified } \\
\text { products } \\
(\mathrm{bp})\end{array}$} & \multirow[b]{2}{*}{$\begin{array}{c}\text { Total } \\
\text { amplicons }\end{array}$} & \multirow[b]{2}{*}{$\begin{array}{l}\text { Total loci } \\
\text { amplified }\end{array}$} & \multicolumn{5}{|c|}{ Origin of loci amplified } & \multirow[b]{2}{*}{${ }^{3}$ Mono } \\
\hline RAPD & $\begin{array}{l}\text { Sequence } \\
5^{\prime}-3^{\prime}\end{array}$ & ISSR & $\begin{array}{c}\text { Sequence } \\
5^{\prime}-3^{\prime}\end{array}$ & & & & $\mathrm{R}^{1}$ & $\mathrm{R}-\mathrm{I}^{1}$ & $\begin{array}{l}\text { R-I } \\
\text { new }\end{array}$ & $\mathrm{R}-\mathrm{I}^{2}$ & $\mathrm{I}^{2}$ & \\
\hline 81 & GAGCACGGGG & 835 & $(\mathrm{AG})_{8} \mathrm{YC}$ & $2180-260$ & 102 & 20 & 10 & 2 & 3 & 3 & 6 & 1 \\
\hline 81 & CCTGGGTGGA & 838 & $(\mathrm{TA})_{8} \mathrm{RC}$ & $1250-250$ & 118 & 22 & 9 & 2 & 5 & 3 & 6 & 2 \\
\hline 84 & GGGCGCGAGT & 811 & $(\mathrm{GA})_{8} \mathrm{C}$ & $1480-230$ & 150 & 24 & 7 & 0 & 4 & 3 & 4 & 6 \\
\hline 85 & GTGCTCGTGC & 838 & $(\mathrm{TA})_{8} \mathrm{RC}$ & $1130-210$ & 114 & 23 & 4 & 1 & 7 & 0 & 8 & 4 \\
\hline 86 & \multirow{3}{*}{ GGGGGGAAGG } & 836 & $(\mathrm{AG})_{8} \mathrm{YA}$ & $1210-220$ & 93 & 17 & 7 & 2 & 5 & 0 & 3 & 2 \\
\hline 86 & & 811 & $(\mathrm{GA})_{8} \mathrm{C}$ & $1330-150$ & 148 & 20 & 7 & 3 & 7 & 2 & 3 & 3 \\
\hline 86 & & 876 & $(\mathrm{GATA})_{2}(\mathrm{GACA})_{2}$ & $1340-210$ & 172 & 25 & 9 & 5 & 8 & 0 & 5 & 3 \\
\hline 89 & GGGGGCTTGG & 836 & $(\mathrm{AG})_{8} \mathrm{YA}$ & $1030-210$ & 105 & 19 & 5 & 2 & 3 & 4 & 7 & 4 \\
\hline 211 & GAAGCGCGAT & 876 & $(\mathrm{GATA})_{2}(\mathrm{GACA})_{2}$ & $1560-270$ & 100 & 19 & 7 & 5 & 7 & 1 & 3 & 3 \\
\hline 215 & TCACACGTGC & 872 & $(\text { GATA })_{4}$ & $1890-280$ & 100 & 17 & 7 & 2 & 3 & 2 & 5 & 2 \\
\hline 226 & GGGCCTCTAT & 835 & $(\mathrm{AG})_{8} \mathrm{YC}$ & $1280-220$ & 97 & 16 & 6 & 0 & 2 & 1 & 6 & 2 \\
\hline 251 & \multirow{2}{*}{ CTTGACGGGG } & 876 & $(\mathrm{GATA})_{2}(\mathrm{GACA})_{2}$ & $2050-350$ & 102 & 26 & 12 & 3 & 8 & 0 & 3 & 3 \\
\hline 251 & & 844 & $(\mathrm{CT})_{8} \mathrm{RC}$ & $2030-380$ & 135 & 22 & 11 & 2 & 3 & 2 & 4 & 5 \\
\hline 284 & CAGGCGCACA & 835 & $(\mathrm{AG})_{8} \mathrm{YC}$ & $1520-280$ & 103 & 17 & 8 & 0 & 2 & 2 & 5 & 2 \\
\hline 302 & CGGCCCACGT & 844 & $(\mathrm{CT})_{8} \mathrm{RC}$ & $1810-200$ & 116 & 20 & 6 & 0 & 4 & 3 & 6 & 4 \\
\hline 338 & CTGTGGCGGT & 835 & $(\mathrm{AG})_{8} \mathrm{YC}$ & $1200-220$ & 105 & 19 & 6 & 1 & 4 & 1 & 3 & 6 \\
\hline 345 & \multirow{2}{*}{ GCGTGACCCG } & 886 & $\operatorname{VDV}(\mathrm{CT})_{7}$ & $1010-270$ & 124 & 14 & 4 & 0 & 2 & 0 & 3 & 5 \\
\hline 345 & & 811 & $(\mathrm{GA})_{8} \mathrm{C}$ & $1130-170$ & 106 & 21 & 8 & 1 & 7 & 0 & 1 & 5 \\
\hline 348 & \multirow{2}{*}{ CACGGCTGCG } & 836 & $(\mathrm{AG})_{8} \mathrm{YA}$ & $1040-190$ & 92 & 20 & 7 & 0 & 7 & 1 & 5 & 1 \\
\hline 348 & & 811 & $(\mathrm{GA})_{8} \mathrm{C}$ & $1410-150$ & 115 & 20 & 11 & 3 & 3 & 2 & 4 & 2 \\
\hline 349 & GGAGCCCCCT & 876 & $(\mathrm{GATA})_{2}(\mathrm{GACA})_{2}$ & $1830-190$ & 118 & 21 & 10 & 5 & 3 & 1 & 6 & 2 \\
\hline 374 & GGTCAACCCT & 876 & $(\mathrm{GATA})_{2}(\mathrm{GACA})_{2}$ & $1270-170$ & 103 & 25 & 9 & 3 & 5 & 0 & 8 & 2 \\
\hline 615 & \multirow{3}{*}{ CGTCGAGCGG } & 872 & $(\text { GATA })_{4}$ & $2000-230$ & 116 & 20 & 14 & 4 & 0 & 2 & 4 & 2 \\
\hline 615 & & 836 & $(\mathrm{AG})_{8} \mathrm{YA}$ & $2070-280$ & 129 & 20 & 10 & 2 & 0 & 3 & 7 & 2 \\
\hline 615 & & 844 & $(\mathrm{CT})_{8} \mathrm{RC}$ & $1960-320$ & 120 & 24 & 6 & 0 & 5 & 0 & 6 & 7 \\
\hline 648 & GCACGCGAGA & 876 & $(\mathrm{GATA})_{2}(\mathrm{GACA})_{2}$ & $1420-270$ & 104 & 19 & 13 & 1 & 1 & 2 & 3 & 2 \\
\hline 693 & \multirow{2}{*}{ GACGAGACGG } & 872 & $\left(\mathrm{GATA}_{4}\right.$ & $2110-170$ & 99 & 20 & 8 & 1 & 5 & 1 & 6 & 1 \\
\hline \multirow[t]{3}{*}{693} & & 811 & $(\mathrm{GA})_{8} \mathrm{C}$ & $1370-220$ & 120 & 17 & 9 & 0 & 1 & 4 & 6 & 2 \\
\hline & & & & & 3206 & 567 & 230 & 50 & 114 & 43 & 136 & 85 \\
\hline & & & & & & & & & 207 & & & \\
\hline
\end{tabular}

${ }^{1} \mathrm{R}$ - RAPD loci; 'R-I - "like-RAPD loci" in R-ISSR profiles; new R-I - loci amplified; ${ }^{2} \mathrm{R}-\mathrm{I}$ - "like-ISSR loci" in R-ISSR profiles; ${ }^{2} \mathrm{I}$ - ISSR loci.

Total number of loci amplified - is sum of ${ }^{1} \mathrm{R}+{ }^{2} \mathrm{R}-\mathrm{I}($ new $)+\mathrm{I}+{ }^{3} \mathrm{Mono} .{ }^{3} \mathrm{Mono}-$ loci observed in RAPD, R-ISSR and ISSR profiles with the same electrophoretic mobility, $\mathrm{Y}=\mathrm{C}+\mathrm{T}, \mathrm{R}=\mathrm{A}+\mathrm{G}, \mathrm{V}=\mathrm{G}+\mathrm{A}+\mathrm{C}, \mathrm{D}=\mathrm{G}+\mathrm{A}+\mathrm{T}$

ing the new genomic loci, depended on their combination with RAPD used in the research. The largest number of new R-ISSR loci were amplified using combinations of 876 (ISSR) and 251 (RAPD), while the fewest (one locus) using 876 (ISSR) + 648 (RAPD) and 811 (ISSR) + 693 (RAPD) primers (Tab. 1).

Sequential analysis of selected amplicons - marked with letters 'a, 'b' and 'd' in Fig. 2A - revealed that, despite identical electrophoretic mobility as 'reference' - RAPD or ISSR products, these constituted the R-ISSR products (Tab. 2A-a, -b, -d). Using the BLAST tool, alignments to sequences of products: 'a', 'b' and 'd' were identified among available est_others and listed in Tab. 2. It was found that the matched sequences were described in various studies as ESTs connected with the response to abiotic stress of various cereals caused by: drought, salinity and aluminium treatments (Tab. 2).
The sequence of the PCR product marked with the letter ' $c$ ' in Fig. 2A was described in the experiment as new genomic R-ISSR loci (Fig. 2A-c, Tab. 2A-c).

Analysis of the sequences of five subsequent amplicons (Fig. 2B, Tab. 2B), including four putative R-ISSRs and one ISSR, revealed that ' $\mathrm{a}$ ' and ' $\mathrm{b}$ ' amplicons were identical R-ISSRs (750 bp long). This product was presented as a molecular marker identified using the BSA method. Product 'a' was identified for the tolerant line - Ot $0-6$ and for bulk (' $b$ ') pool of the DNAs of twenty tolerant RILs $\left(\mathrm{F}_{9}\right)$. This product was not detected for inbred line Ot $1-3, \mathrm{~F}_{1}$ or in a susceptible bulk (Fig. 2).

Tab. 3 presents a contig created as a result of assembling sequences of a single DNA strand obtained for the 'a' and 'b' inserts (Tab. 2 and 3).

High e-values of alignments to sequences of the RISSR marker (R-I_85+836_750) for various ESTs were 
242

Tab. 2. PCR product sequencing result of selected amplicons - described as results of R-ISSR or ISSR - and the result of their NCBI base sequencing alignments (EST)

Fig. 2 Product Sequence
Type of product and length bp

\begin{tabular}{cc}
\hline & \\
A & a \\
Accession E-value
\end{tabular}

cap_MF30a GATAGATAGA CAGACAAGAA GGTGGTTAAG cap_MF30e GATAGATAGA CAGACAAGAA GGTGGTTAAG Cap_MR30a $\ldots \ldots \ldots \ldots \ldots \ldots \ldots \ldots \ldots \ldots \ldots$

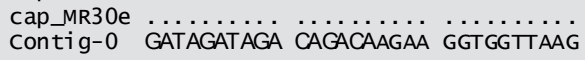
cap_MF30a $\ldots \ldots \ldots \ldots \ldots \ldots \ldots \ldots \ldots \ldots$

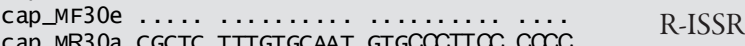
cap_MR30e CGCTC TTTGTGCAAT GTGCOCTTœ COCC 816 bp

R-ISSR
816 bp

\begin{tabular}{lll}
\multicolumn{3}{c}{ and drought stressed shoots and crowns 2e-37); callus (barley: BU993554.1 - 2e-06 transcriptom analysis) } \\
b align_MF32a GGGGGAAGG CCGACGGGGA CGGGTATCAA & align_MF32a TGTG CCATGTCATC TGTCTGTCTA TCTATC \\
align_MF32c GCGGGGAGG CCGACGGGG CGGGTATCAA & align_MF32c TGTG CCATGTCATC TGTCTGTCTA TCTATC
\end{tabular}

Accession E-value

c align_MF33c GATAGATAGA CAGACAAT TAGGCTGTC align_MF33a GATAGATAGA CAGACAAT TAGGCTGTC callus: barley (BQ659305.1 - 2e-77, transcriptom analysis); identification of stress response genes (wheat: CJ523291.1 - 6e-77), genomics of abiotic stress (wheat and canola: CK207925.1 - 2e-23) $\begin{array}{ll}\text { align_MF33C TCACCG GGTTCATCT GACTGCCTTC COCOC } & \text { R-ISSR } \\ \text { align_MF33a TCACCG GGTTCATCT GACTGCCTTC COCOC } & 365 \text { bp }\end{array}$ not found
Accession E-value

cap_MF31d GGGGGGAAGG GCACACATCG CTGGTCGCCG cap_MF31b GCGGGGAAGG GCACACATCG CTGGTCGCCG cap_MR31d $\ldots \ldots \ldots \ldots \ldots \ldots \ldots \ldots \ldots \ldots$.

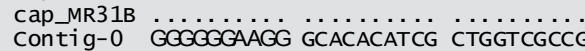

cap_MF31d cap cap_MR31B CTTTCCTC CTCGTATGTC TGTCTATCTA TC contig-0 CTTTCCTC CTCGTATGTC TGICTATCTA TC

Accession E-value treatments: aluminium (wheat (root): CJ870080.1, CJ865897.1, CJ864508.1, CJ853125.1 - 2e-14)

ISSR - (5'GATA GATA GACA GACA 3`) + RAPD - ('3 GGAAGGGGGG 5’) or RAPD (5’ GGGGGGAAGG 3’) + ISSR - (3’ACAG ACAG ATAG ATAG 5’)
cap_MF3a GTGCTCGTG CATACGCCAG GTACTTATCA cap_MF3b GTGCTCGT GC CATACGCCAG GTACTTATCA cap_MR3b $\ldots \ldots \ldots \ldots \ldots \ldots \ldots \ldots \ldots \ldots \ldots$

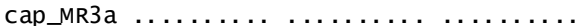
contig-0 GT

cap_MF4f GT CTCGT $\subseteq$ CATACGCCAG GTACTTATCA cap_MF4g GT@TCGT $@$ CATACGCCAG GTACTTATCA cap_MR4g $\ldots \ldots \ldots \ldots \ldots \ldots \ldots \ldots \ldots \ldots$

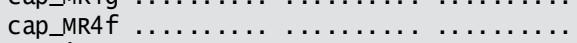

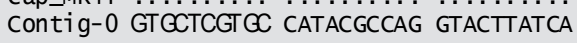
сар $\_$MF3a $\ldots \ldots \ldots \ldots \ldots \ldots \ldots$

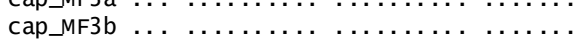

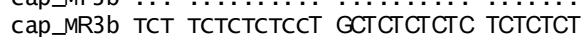 cap_MR3a TCT тстстстCCT АCTCTCTCTC TCTCTCT R-ISSR contig-0 TCT TCTCTCTCCT ACTCTCTCTC TCTCTCT
cap_MR4f тCTTCTC тCTCCTACTC TCTCTCTCTC TCT cap_MR4g TCTTCTC TCTCCTACTC TCTCTCTCTC TCT

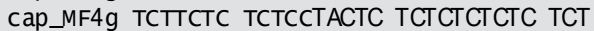 cap_MF4f TCTTCTC TCTCCTACTC TCTCTCTCTC ТCT contig-0 TCTTCTC TCTCCTACTC TCTCTCTCTC TCT
R-ISSR
$750 \mathrm{bp}$

treatments: aluminium (wheat: CJ808093.1 - 2e-45 - unpublished), cold (rye: BE704490.1 - 4e-11, unpublished), drought (barley: BG300360.1 - 7e-27, CD662726.1 - 1e-18 - unpublished), salinity (wheat: CK197361.1 - 1e18 - unpublished). Project 'Genoplante' (wheat: CD877496.1 - 6e-66, CD881787.1 - 1e-42, CD891252.1 - 3e-

Accession E-value 18, CD875216.1 - 3e-18 - unpublished) - "Validation of gene function to increase the agricultural productivity of wheat and their tolerance to i.a. climatic variation". SCARECROW-like GRAS-family - transcription factor (TF) - candidate gene - Leymus sp. - wild rye: EG393386.1 - 1e-35, EG377967.1 - 3e-32 - Kaur et al. 2008); screening of stress response genes in wheat (CJ682864.1-2e-14, CJ576180.1 - 93-13 - Mochida et al. 2006)

cap_MR5d AGAGAGAGAG AGAGAGACA CGTATGCGCA cap_MF5d ....................... Contig-0 AGAGAGAGAG AGAGAGCACA CGTATGCGCA
cap_MR5d GCAC TTCAGGATTA CTCTCTCTCT CTCTCT cap_MF5d GCAC TTCAGGATTA CTCTCTCTCT CTCTCT contig-0 GCAC TTCAGGATTA CTCTCTCTCT CTCTCT $\underline{\text { ISSR }}$ $357 \mathrm{bp}$

Accession E-value not found

d cap_MF6a AGAGAGAGAG AGAGAGTACA GTCTGTTTCG cap_MR6a AGAGAGAGAG AGAGAGTACA GTCTGTTTCG contig-0 AGAGAGAGAG AGAGAGTACA GTCTGTTTCG

cap_MF6a СTACT GCTGCTTGGT GCTCTCTCTC TCTCTCT cap_MR6a CTACT GCTGCTTGGT GCTCTCTCTC TCTCTCT contig-0 CTACT GCTGCTTGGT GCTCTCTCTC TCTCTCT

ISSR $367 \mathrm{bp}$

Accession E-value expression profile of storage proteins gene families (wheat: BJ273720.1); wheat (anthers ungergoing meiosis) CA599965.1

cap_MF7c AGAGAGAGAG AGAGAGCACG TGCTCGTCGT cap_MF7d AGAGAGAGAG AGAGAGCACG TGCTCGTCGT cap_MR7c AGAGAGAGAG AGAGAGCACG TGCTCGTCGT cap_MR7d AGAGAGAGAG AGAGAGCACG TGCTCGTGGT contig-0 AGAGAGAGAG AGAGAGCACG TGCTCGTCGT
cap_MF7C CT CTAGCCAACC CTACGTGAGC ACGAGCAC cap_MF7d CT CTAGCCAACC CTACGTGAGC AOGAGCAC cap_MR7c CT CTAGCCAACC CTACGTGAGC AOGAGCAC cap_MR7d CT CTAGCCAACC CTACGTGAGC AOGAGCAC Cap_MR7d CT CTAGCCAACC CTACGTGAGC AOGAGCAC
contig-0 CT CTAGCCAACC CTACGTGAGC AOGAGCAC
R-ISSR $258 \mathrm{bp}$

Accession E-value treatments: drougth - screening of stress response genes (wheat: CJ717079.1, CJ612834.1)

ISSR - 5' GATA GATA GACA GACA 3’ + RAPD ('3 GGAAGGGGGG 5’) or RAPD - (5' GTGCTCGTGC 3’) + ISSR - (3’ AY8[GA] 5’) 
Tab. 3. Sequences and fingerprints of PCR product (R-ISSR marker) identified for inbred line and bulk tolerant to nutrient deficiencies

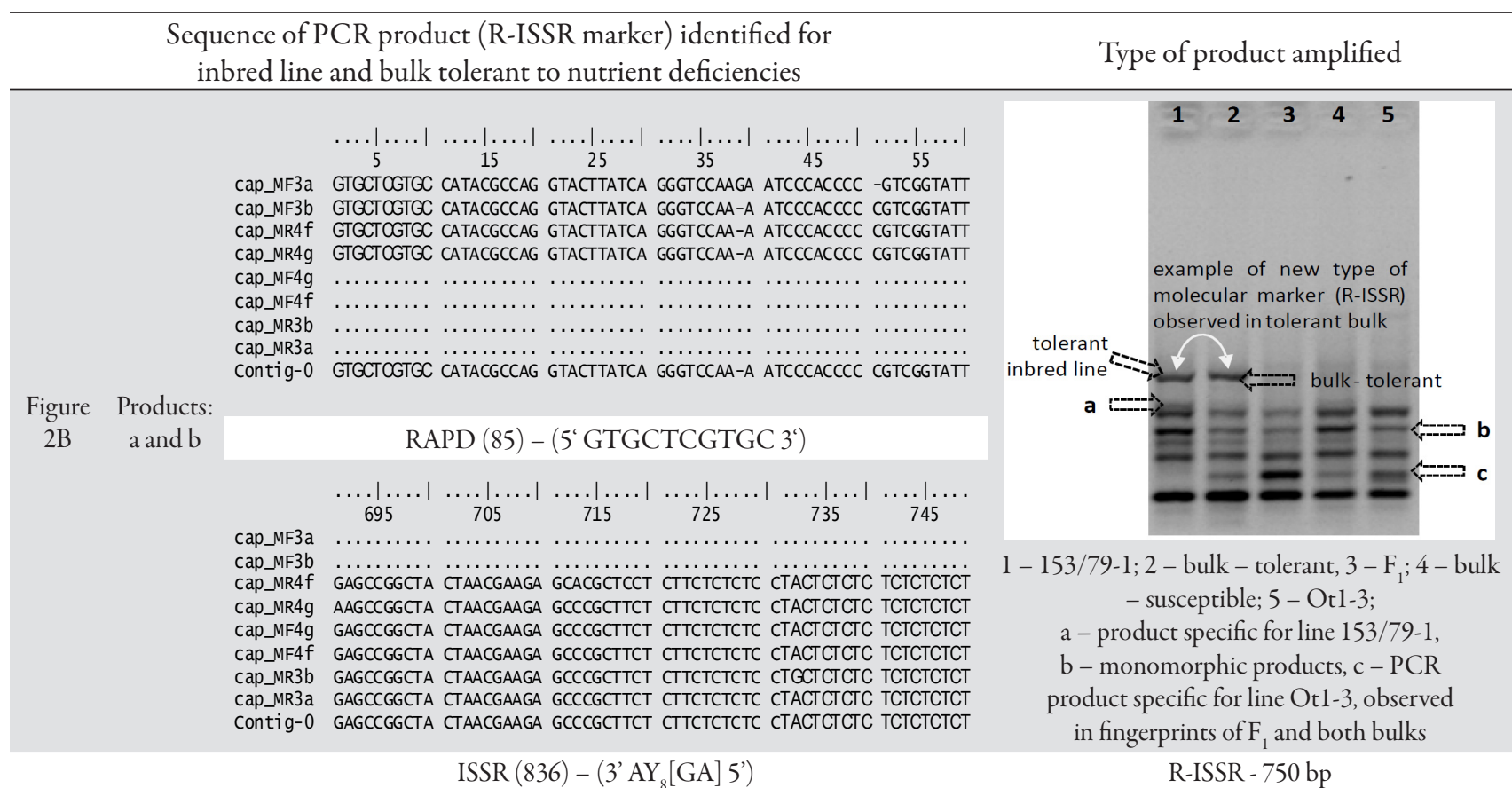

confirmed, including: wheat, barley, Leymus sp. and rye, which were obtained during research on the construction of cDNA libraries, in which mRNA was harvested from different plant organs affected by stress induced by various treatments, i.e. drought, aluminium, salinity and cold. Selected records are presented in Tab. 2.

The amplification of 20 tolerant (RILs) and 20 susceptible DNA samples revealed that the R-ISSR product showed frequency deviated from the expected 1:1 ratio established on the basis of the $\chi^{2}$ test. Frequency within the group tolerant to nutrient deficiency stress (band:null) was 17:3 $\left(9.80^{* *}\right)$, whereas within the group of susceptible RILs (band:null) it amounted to 7:13 (0.20) (Fig. 3).

The ' $\mathrm{e}$ ' product was described as a new genomic R-ISSR locus, whereas 'c' as ISSR (Tab. 2, Fig. 2B, Tab. 2B-c).
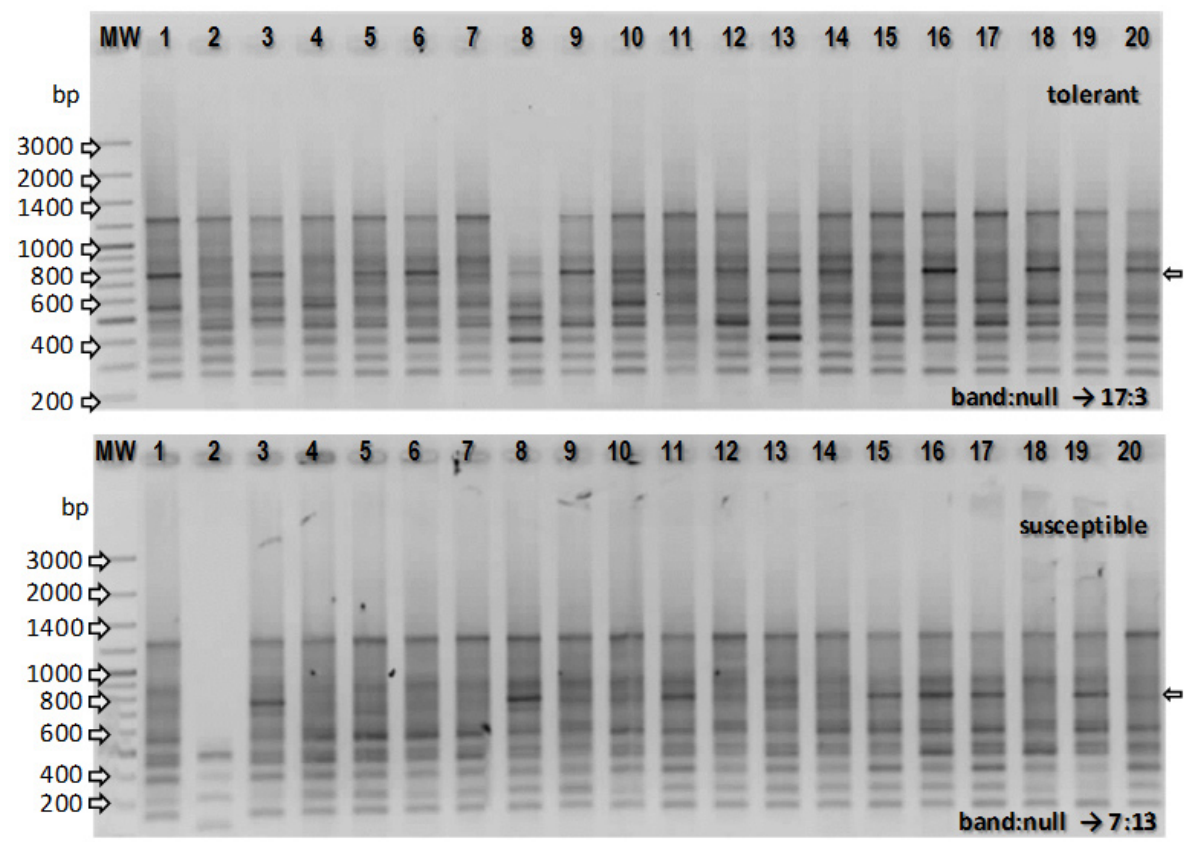

Fig. 3. Electrophoregrams of R-ISSR fingerprints presenting segregation of the R-ISSR marker $\left(R-I_{-} 85+836\right.$-750) in two groups of RILs of rye differing in response to nutrient deprivation stress. Differences in segregation (band:null ratio) were established on the basis of the $\chi 2$ test 
244

Assessment of genetic similarity between rye genotypes was presented in the form of a dendrogram (Fig. 4).

Each of these groups shows genetic relationships between rye genotypes analyzed using one of three different methods. Genotypes characterized according to R-ISSR fingerprints were included in group 'a', ISSR in group ' $b$ ', whereas RAPD in group 'c'. A significant correlation $\left(r_{A B . C}\right.$ $=0.964)$, calculated by means of the Mantel test, indicated congruence between the compared matrices and, in this case, molecular marker methods.

\section{Discussion}

The results of this study confirm the possibility of using the R-ISSR tool for exploring the new scope of variability in the rye genome occurring in informative and poorly known regions, and microsatellite and inter-simple sequence repeat sequences, which were not often amplified due to limitations, e.g. primer hybridization sites, screening methods, i.e. RAPD (Ćwiklińska et al., 2010; Myśków et al., 2001) and ISSR, as well as SSR and AFLP (Lawson and Zhang, 2006; Rakoczy-Trojanowska and Bolibok, 2004; van der Nest et al., 2000; Ye et al., 2005).

As opposed to Ye et al. (2005) who first used 13-14 bps long ISSR primers in R-ISSR, 16-18 bps long ISSR were used in this research, in a manner similar to Saleh (2011). Those with low $\mathrm{T}_{\mathrm{m}}$ were selected from amongst them and were combined with selected RAPD primers in multiplex PCR.

R-ISSR products, as in the studies of Ye et al. (2005) and Saleh (2011), were separated in 1.5\% agarose gel. RISSR fingerprints obtained for two hybrid lines, $\mathrm{F}_{1}$ and for two bulks were described as repeatable and co-dominant R-ISSR products. Both features, as opposed to RAPD, are characteristic of ISSR (Pradeep Reddy et al., 2002; Rakoczy-Trojanowska and Bolibok, 2004).

A set of R-ISSR products was amplified and described in the experiment, in a manner similar to Ye et al. (2005) and Saleh (2011). As shown in Tab. 1, 50 products were described as 'like'-RAPD, 43 as 'like'-ISSR, and 114 as new

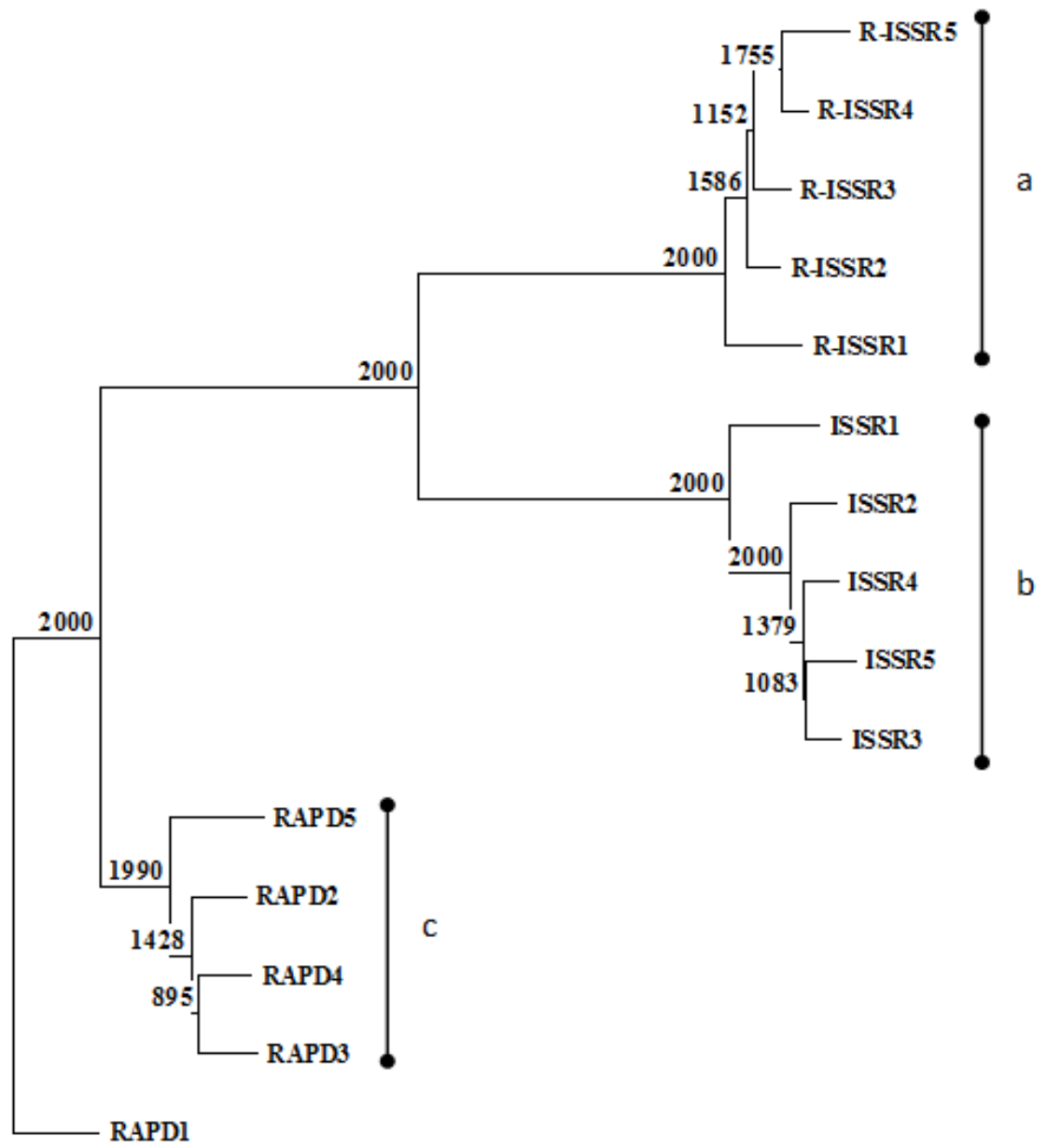

a, b and c-groups of similarity

1 - Ot0-6; 2 - bulk - RILs tolerant to nutrient deprivation; 3 - $\mathrm{F}_{1}(\mathrm{Ot} 0-6 \times \mathrm{Ot} 1-3) ; 4$ - bulk RILs susceptible to nutrient deprivation; 5 - Ot1-3

Fig. 4. UPGMA dendrogram presenting genetic relationships among five rye genotypes investigated via three different techniques (RAPD, R-ISSR and ISSR). Numbers above branches indicate the real values of bootstraps in which the branch was observed in 2,000 pseudoreplications 
R-ISSR. In sequential analysis, it was shown that three RISSR products, which, following Ye et al. (2005) were regarded as 'identically' migrating as RAPD and ISSR constituted R-ISSR products (Fig. 2A, a, b, d). Two out of the selected R-ISSR amplicons were sequenced. It was shown that they were R-ISSR products (Fig. 2A-c, B-e).

These results modify the opinion presented by Ye et al. (2005), which was later adopted by Saleh (2011). The results provide new information concerning the essence of R-ISSR. It makes it possible to conclude that three types of products should be obtained in multiplex PCR while using RAPD and ISSR primers: ISSR, RAPD and heteroamplicons - R-ISSR. It was confirmed that 'typical' R-ISSRs have a heteroamplicon character; however, RISSR products co-migrating as RAPD and ISSR are rarely RAPD and ISSR. They are usually R-ISSR with lengths differing by several base pairs. It was shown that one R-ISSR product (Fig. 2B-c) was an ISSR with a length similar to that 'referentially' sequenced.

Not all products present in the R-ISSR profiles were sequenced; hence, conclusions are limited only to the results obtained for sequences of seven amplicons selected from two different R-ISSR fingerprints which presented the essence of R-ISSR. The sequential character of 85 R-ISSR products migrating in agarose gel with the same mobility as RAPDs and ISSRs was not described (Tab. 1). They were found in R-ISSR fingerprints and described by Ye et al. (2005) and Saleh (2011). It can only be supposed that these are heteroamplicons of a similar length.

In the profiles of the two bulks presented in this study (Fig. 2B - R-ISSR), one R-ISSR product specific for tolerance to nutrient deprivation inbred lines - Ot0-6 was identified. This product was considered to be a molecular marker putatively linked to one of the loci determining rye tolerance to nutrient deficiencies. Sequential analysis and sequence alignment performed using BLAST has shown its high homology to various ESTs expressed in response to abiotic stress caused by different treatments: aluminium, drought, salinity and cold, where the criterion of morphological assessment of response to stress is, amongst others, root morphology (Tab. 2). One of the ETS sequences aligned to the sequence of the R-ISSR marker was the locus of the SCARECROW-like GRAS-family transcription factor (TF) (BG01_2.3579.C1.Contig4507) (1e-35) identified amongst wild rye ESTs (Leymus sp. - Triticeae) by Kaur et al. (2008). This transcription factor is involved in cell division, but is not sufficient for cell differentiation. The $S C R$ mRNA was localized in the root within the cortex/endodermis zone in Arabidopsis (Di Laurenzio et al., 1996). ETS no. CJ808093.1 (2e-45) is another interesting sequence. It was identified during a project by Ogihara et al., (unpublished - deposed in 2007 in GenBank) as results of a transcriptome analysis (cDNA) of wheat roots growing in aluminium treated soil. This response leads to a dramatic reduction in root growth (Aniol, 1990; Milla et al., 2002).

Without a comprehensive analysis of data obtained from research on the rye transcriptome (ESTs, microar- rays), it is difficult to match the biological functions to an individual marker (sequence). Information about the sequence of one (or more) molecular marker(s) occur within bulks, in accordance with one possible pattern presented by Michelmore et al. (1991), Masojć et al. (2009), which may confirm the usefulness of R-ISSR in the identification of pools of putative molecular markers. These experiments need to be continued with an attempt at determination of their chromosomal localization and thereby confirmation of their usefulness as an additional type of markers used for construction and saturation of genetic maps.

The correlation coefficient calculated using the Mantel test for RAPD, R-ISSR and ISSR matrices was high $\left(r_{A B C}=0.964\right)$ and may indicate congruence between the compared molecular marker methods (Sneath and Sokal, 1973).

\section{Conclusions}

R-ISSR is a cost-efficient research tool and can be used, alongside RAPD, ISSR or other tools like IRAP REMAP (Kalendar and Schulman, 2006; Smykal, 2006) for evaluation of genetic variability, fingerprinting, identification of molecular markers, and mapping for gene tagging not only for rye. It has been demonstrated that already a few ISSR primers are capable, together with various RAPDs, to generate informative R-ISSR products providing a new set of biological information.

\section{Acknowledgements}

This work was supported by the Polish Ministry of Science and Higher Education under grant No. N N302 281936.

I am grateful to my colleagues Paweł Milczarski, D.Sc. and Radosław Owsianicki, M.Sc. for excellent discussions about technical solutions concerning cloning and sequencing protocols.

\section{References}

Akhavan A, Saeidi M, Rahiminejad MR (2010). Genetic diversity of Secale cereale L. in Iran as measured using microsatellites. Genet Res Crop Evol 57:415-422.

Anioł A (1990). Genetics of tolerance to aluminum in wheat (Triticum aestivum L. Thell). Plant Soil 123:223-227.

Bartoš J, Paux E, Kofler R, Havránková M, Kopecký D, Suchánková P, Šafár J, Šimková H, Town ChD, Lelley T, Feuillet C, Doležel J (2008). A first survey of the rye (Secale cereale L.) genome composition through BAC end sequencing of the short arm of chromosome 1R. BMC Plant Biology BMC 8:95 doi:10.1186/1471-2229-8-95.

Bednarek PT, Masojć P, Lewandowska R, Myśków B (2003). Saturating rye genetic map with amplified fragment length polymorphism (AFLP) and random amplified polymorphic DNA (RAPD) markers. J Appl Genet 44(1):21-33.

Bolibok H, Rakoczy-Trojanowska M, Wyrzykowska M, Radecka M, Orczyk W (2006). Identification of microsatellite markers in the rye genome. Cell Mol Biol Lett 11:291-298. 
246

Buntjer JB (2001). PHYLTOOLS Phylogenetic Computer Tools V. 1.32 for Win. Wageningen Agricultural University, 2001, The Netherlands.

Ćwiklińska A, Broda Z, Bocianowski J, Dobrzycka A (2010). The usefulness of RAPD and AFLP markers for determining genetic similarity in rye (Secale L.) species and subspecies. Acta Biol Cracov Series Botanica 52(1):19-25.

Devos KM, Gale MK (2000). Genome relationships: The grass model in current research. Plant Cell 12:637-646.

Di Laurenzio L, Wysocka-Diller J, MalamyJE, Pysh L, Helariutta Y, Freshour G, Hahn MG, Feldmann KA, Benfey PN (1996). The SCARECROW gene regulates an asymmetric cell division that is essential for generating the radial organization of the Arabidopsis root. Cell 86:423-433.

Flavell RB, Bennett MD, Smith JB, Smith DB (1974). Genome size and the proportion of repeated nucleotide sequence DNA in plants. Biochem Genet 12(4):257-269.

Geiger HH, Miedaner T (2009). Rye breeding, 157-181 p. In: Carena MJ (Ed.). Cereals, Springer-Science + Business Media., LLC 2009, doi: 10.1007/978-0-387-72297-9.

Hillel D, Rosenzweig C (2005). The role of biodiversity in agronomy. Adv Agron 88:1-34.

Kalendar R, Schulman AH (2006). IRAP and REMAP for retrotransposon-based genotyping and fingerprinting. Nat Protoc 1(5):2478-2484.

Kaur P, Larson SRB, Bushman S, Wang RRC, Mott IW, Hole D, Thimmapuram J, Gong G, Liu L (2008). Genes controlling plant growth habit in Leymus (Triticeae): maize barren stalk1 (bal), rice lax panicle, and wheat tiller inbibition (tin3) genes as possible candidates. Funct Integr Genomics 8:375-386.

Lawson MJ, Zhang L (2006). Distinct patterns of SSR distribution in the Arabidopsis thaliana and rice genomes. Genome Biol 7(2):R14.

Liu J, Han L, Chen F, Bao J, Zhang F, Mi G (2008). Microarrays analysis reveals early responsive genes possibly involved in localized nitrate stimulation of lateral root development in maize (Zea mays L.). Plant Sci 175:272-282.

Masojć P, Lebiecka K, Milczarski P, Wiśniewska M, Łań A, Owsianicki R (2009). Three classes of loci controlling preharvest sprouting in rye (Secale cereale L.) discerned by means of bidirectorial selective genotyping (BSG). Euphytica 170:123-129.

Michelmore RW, Paran I, Kesseli RV (1991). Identification of markers linked to disease-resistance genes by bulked segregant analysis: A rapid method to detect markers in specific genomic regions by using segregating populations. PNAS 88:9828-9832.

Milla MAR, Butler E, Huete AR, Wilson CF, Anderson O, Gustafson JP (2002). Expressed sequence tag-based gene expression analysis under aluminium stress in rye. Plant Physiol 130:1706-1716.

Mochida K, Kawaura K, Shimosaka E, Kawakami N, Shin-I T, Kohara Y, Yamazaki Y, Ogihara Y (2006). Tissue expression map of a large number of expressed sequence tags and its application to in silico screening of stress response genes in common wheat. Mol Gen Genomics 276:304-312.

Moller ALB, Pedas P, Andersen B, Svensson B, Schjoerring JK, Finnie Ch (2011). Responses of barley root and shoot proteomes to long-term nitrogen deficiency, short-term nitrogen starvation and ammonium. Plant Cell Environ 34(12):2024-2037.

Myśków B, Masojć P, Banek-Tabor A, Szołkowski A (2001). Genetic diversity of inbred rye lines evaluated by RAPD analysis. J Appl Genet 42:1-14.

Pradeep Reddy M, Sarla N, Siddiq EA (2002). Inter simple sequence repeat (ISSR) polymorphism and its application in plant breeding. Euphytica 128:9-17.

Rakoczy-Trojanowska M, Bolibok H (2004). Characteristics and a comparison of three classes of microsatellite-based markers and their application in plants. Cell Mol Biol Lett 9:221-238.

Rzepka-Plevneš D, Kurek J (1997). The tolerance of some rye varieties and breeding strains to nutrition deficiency using in vitro cultures. Plant Breed Seed Sci 41:41-49.

Saleh B (2011). R-ISSR marker as a useful tool for detection of new genomic loci in Arthrocnemum macrostachyum. Biol Plant 55(2):327-330.

Smykal P (2006). Development of an efficient retrotransposonbased fingerprinting method for rapid pea variety identification. J Appl Genet 47(3):221-230.

Sneath P, Sokal R (1973). Numerical taxonomy: The principles and practice of numerical classification. San Francisco., CA: W. H. Freeman.

Van de Peer Y, De Wachter R (1994). TREECON for Windows: a software package for the construction and drawing of evolutionary trees for the Microsoft Windows environment. Comp Appl Biosci 10:569-570.

van der Nest MA, Steenkamp ET, Wingfield BD, Wingfield MJ (2000). Development of simple sequence repeat (SSR) markers in Eucalyptus from amplified inter-simple sequence repeats (ISSR). Plant Breed 119:433-436.

Williams JGK, Kubelik AR, Livak KJ, Rafalski JA, Tingey SV (1990). DNA polymorphisms amplified by arbitrary primers are useful as genetic markers. Nucl Acids Res 18:6531-6535.

Wolfe MS, Baresel JP, Desclaux D, Goldringer I, Hoad S, Kovacs G, Löschenberger F, Miedaner T, Ostergard H, Lammerts van Bueren ET (2008). Developments in breeding cereals for organic agriculture. Euphytica. 163:323-346.

Xu Z, Wei W, Gagneur J, Clauder-Münster S, Smolik M, Huber W, Steinmetz LM (2011). Antisense expression increases gene expression variability and locus interdependency. Mol Syst Biol 7(468) doi:10.1038/msb.2011.1

Ye C, Yu Z, Kong F, Wu S, Wang B (2005). R-ISSR as a new tool for genomic fingerprinting, mapping, and gene tagging. Plant Mol Biol Rep 23:167-177.

Zietkiewicz E, Rafalski A, Labuda D (1994). Genome fingerprinting by simple sequence repeat (SSR)-anchored polymerase chain reaction amplification. Genomics 20:176183. 